Revue

Revue de l'histoire des religions

de Ihistoire

des religions

$3 \mid 2015$

Varia

Sara MATTEOLI, Alle origini della teologia di Pelagio. Tematiche e fonti delle Expositiones XIII epistularum Pauli

Pisa - Roma, Fabrizio Serra editore (« Studi sulla Tardoantichità », 6), 2011

Jérémy Delmulle

\title{
OpenEdition
}

Journals

Édition électronique

URL : http://journals.openedition.org/rhr/8430

DOI : 10.4000/rhr.8430

ISSN : 2105-2573

Éditeur

Armand Colin

Édition imprimée

Date de publication : 1 septembre 2015

Pagination : 440-443

ISBN : 978-2-200-92993-0

ISSN : 0035-1423

Référence électronique

Jérémy Delmulle, "Sara matteol, Alle origini della teologia di Pelagio. Tematiche e fonti delle Expositiones XIII epistularum Pauli », Revue de l'histoire des religions [En ligne], 3 | 2015, mis en ligne le 21 octobre 2015, consulté le 25 septembre 2020. URL : http://journals.openedition.org/rhr/8430 ; DOI : https:// doi.org/10.4000/rhr.8430

Ce document a été généré automatiquement le 25 septembre 2020.

Tous droits réservés 


\section{Sara MATTEOLI, Alle origini della teologia di Pelagio. Tematiche e fonti delle Expositiones XIII epistularum Pauli}

Pisa - Roma, Fabrizio Serra editore (« Studi sulla Tardoantichità », 6), 2011

Jérémy Delmulle

\section{RÉFÉRENCE}

Sara MATtEoLI, Alle origini della teologia di Pelagio. Tematiche e fonti delle Expositiones XIII epistularum Pauli, Pisa - Roma, Fabrizio Serra editore (« Studi sulla Tardoantichità », 6), 2011, 25 cm, 200 p., $48 €$, ISBN : 978-88-6227-370-1.

« Pélage, réformateur et novateur » : c'est sous ce titre que G. de Plinval, dans une thèse qui a fait date (Pélage. Ses écrits, sa vie et sa réforme, Lausanne 1943), étudiait l'influence déterminante de Pélage sur les penseurs de son temps et ceux qui allaient suivre. Cette image, incontestable, d'innovation ne doit pourtant pas occulter la signification première de "réforme » et les différentes dettes que le moine breton a contractées auprès de ses devanciers. Cela est d'autant plus vrai pour la partie proprement exégétique de son œuvre - son «Commentaire à Paul» - pour laquelle Pélage ne pouvait que se placer au sein d'une tradition déjà bien ancrée, chez les Grecs et dans l'Occident latin. Plinval posait déjà le problème en des termes lumineux (p. 121-166). Mais depuis les années 1980-1990, grâce surtout aux publications de Th. De Bruyn sur les Expositiones, qui revenaient sur les travaux de Souter (1922-1926), le terrain était devenu suffisamment balisé pour que l'on reprît plus sûrement ce dossier. C'est ce à quoi s'est attelée S. Matteoli dans une thèse soutenue en 2010 et publiée dès l'année suivante. 
2 Dans une brève introduction, qui vise surtout à dresser le bilan des recherches menées sur ce texte assez méconnu, l'A. rappelle commodément le contexte doctrinal qui a présidé à la composition (vers 406-409) de ce commentaire, en le rattachant aux débats exégétiques entretenus alors avec les manichéens. C'est dans le premier chapitre que sont présentées les quatre sources principales identifiées dans le commentaire: Origène, l'Ambrosiaster, l'Anonyme de Budapest et Augustin. Cinq chapitres forment ensuite le corps de l'ouvrage, dont chacun entend traiter un aspect particulier de la théologie des Expositiones. Dans le deuxième chapitre, qui s'intéresse à la notion de nature dans le commentaire pélagien, l'A. souligne les nombreux points de contact de la pensée de Pélage avec celle de l'Ambrosiaster, mais également celle de Rufin le Syrien, de Tertullien ou encore - quoique leurs rapports soient plus complexes - celle d'Origène. C'est plus particulièrement le concept de lex naturae qui, outre les échos qu'il trouve dans la tradition stoïcienne et chez les auteurs chrétiens précédant Pélage, voit chez ce dernier, et dans ses développements sur la valeur des justes de l'Ancien Testament, se dessiner son expression la plus originale : dès les Expositiones, l'ascète breton soutient qu'en vertu de cette loi naturelle, l'homme disposerait d'une force intérieure et de capacités suffisantes pour se conduire correctement.

3 Le chapitre suivant, le plus long de l'ouvrage, est tout entier dédié au problème du péché d'Adam, dont l'examen est d'autant plus important que la question de la nature et des conséquences de la faute originelle était, selon Augustin, une pierre d'achoppement entre Pélage et Célestius. Recherchant dans le commentaire à Paul la formulation de certaines positions qui auraient pu donner naissance aux idées ultérieurement défendues par Pélage, l'A. les compare surtout avec la manière dont le sujet est traité par tous les prédécesseurs de Pélage, tant grecs que latins, et en s'attardant même sur le cas d'Augustin. Si la pensée de Pélage peut puiser certains éléments de sa conception du péché et de sa transmission à la pensée grecque, comme pour le thème précédent, le rapport qu'il entretient plus spécifiquement avec la pensée d'Origène est fait de dette et d'originalité.

Le libre arbitre fait lui aussi l'objet d'une étude particulière (ch.4), dans laquelle l'A. examine l'un après l'autre les concepts-clés (impeccantia, prédestination et grâce divine) et les principaux passages pauliniens (surtout Rom. 7 et 9) sur lesquels, durant la controverse, Pélage appuiera sa théorie personnelle sur la valeur et le rôle de la volonté humaine. Ce faisant, Pélage se place tout à fait dans la continuité de la pensée d'Origène qui, examinant déjà les mêmes passages, en soulignait la difficulté et mettait surtout en garde contre une mélecture qui annihilerait le pouvoir de la liberté : à son tour, et donc avant que n'éclate la controverse proprement dite, Pélage éprouve la nécessité d'insister sur la liberté de la volonté. C'est cette question même de la liberté qui amène l'A. à consacrer un excursus au De induratione cordis Pharaonis (p. 145-156), dans lequel elle suggère de voir l'œuvre d'un disciple de Pélage, qui l'aurait composé après 411 voire, dit-elle, après 418.

Quant au cinquième chapitre, consacré à la grâce et aux questions de la justification et du salut (p. 157-173), il scrute d'abord les diverses significations du mot gratia, dont il faut remarquer qu'il sert, dans les Expositiones, à désigner à la fois un don gratuit et la récompense de mérites. Malgré cette contradiction apparente, Pélage insiste sur son absolue nécessité et son entière gratuité. Ce sont davantage des visées parénétiques qui poussent Pélage à mettre l'accent sur les œuvres, sans lesquelles on perdrait le bénéfice de la grâce octroyée. En développant cette théorie de la grâce, atteignable grâce à ce 
commentaire par un biais qui ne soit pas le prisme augustinien, Pélage apparaît, vis-àvis des positions de ses prédécesseurs, comme un conciliateur cherchant à faire correspondre des idées a priori antithétiques, plus que comme un véritable innovateur.

6 Le dernier chapitre recherche, quant à lui, les traces d'une présence dans les Expositiones du concept-clé d'impeccantia, indissociablement lié à Pélage (quoique le mot ait été forgé par Jérôme). Si cette conception pélagienne peut remonter, comme du reste Jérôme lui-même le prétendait, à l'apatheia stoïcienne, elle a aussi plus directement subi l'influence d'Origène, de Rufin et de Sextus. Car généralement pour les Pères, apatheia, anamartesia et impeccantia ne peuvent concerner que Dieu et le Christ. Pour les pélagiens, l'impeccantia n'est que l'expression d'une possibilité théorique et n'a de visée que parénétique : l'appel à la perfection lancé par Pélage ne doit pas être confondu avec une réalité historique, mais est à prendre dans le sens (très ambrosien) que l'homme, une fois converti, pourrait avoir la possibilité de ne plus pécher. Quant à définir quel est le discours tenu là-dessus dans les Expositiones, c'est quasiment impossible, vu la rareté des passages concernés (due au genre littéraire choisi ou au désintérêt relatif de Pélage à cette époque ?) et surtout aux profonds désaccords attestés dans la tradition textuelle.

7 Avec un tel objet d'étude, il n'était pas facile d'éviter les défauts de structure. Consacrer un chapitre à chaque auteur-source pour mesurer le degré de dépendance contracté par Pélage à son égard, ou choisir tour à tour un sujet majeur et passer en revue, à chaque fois, les rapports de Pélage sur la question avec les écrits de ses devanciers? L'une et l'autre solution comportait le risque de répétitions ou d'un discours morcelé. Mais la première aurait peut-être eu l'avantage de souligner des thématiques plus inattendues ou des éclairages différents. Car, quoique l'auteur prétende (p. 189) prendre l'Expositio comme point de départ et tirer de sa lecture les idées les plus saillantes, et comme plusieurs formulations (p. $48,55,117)$ tendent à le suggérer, les grands thèmes développés dans chacun des chapitres sont fortement influencés par la connaissance rétroactive des idées qui formeront, lors de la controverse, la doctrine pélagienne : rapports entre nature et loi, question de la foi, du péché originel, notion d' impeccantia, etc. Cette recherche quelque peu orientée nuit donc, en un sens, à la démonstration, et empêche qu'une réponse claire soit apportée à la question de départ, qui est l'important : Pélage était-il pélagien avant le pélagianisme? Mais elle permet, $\mathrm{du}$ moins, de bien comprendre quels sont les véritables apports personnels de sa pensée, ce qui serait hérité de telle ou telle source identifiée et ce qui n'appartient, au fond, qu'à un substrat totalement étranger à ce qui deviendra le pélagianisme et qui aura pu influencer également d'autres pensées.

8 Mais l'embarras de la réponse, concernant la genèse de la pensée pélagienne, tient aussi aux difficultés mêmes de la source choisie. Rien n'est moins simple que de se faire une idée juste d'une pensée qui n'est atteignable, comme c'est le cas ici, qu'à travers des annotations parfois très brèves et des gloses ponctuelles qui ne suivent d'autre ordre que celui du texte paulinien. L'établissement même du texte n'est pas sans poser des problèmes de taille: plusieurs variantes de fond, qui plus est volontaires, viennent compliquer des passages touchant précisément les sujets de la grâce, du libre arbitre, de l'impeccantia, dans les deux témoins principaux, $A$ et $B$. On n'a pu encore établir si c'est $A$ qui donne une version édulcorée du texte de Pélage ou si c'est $B$ qui transmet un texte au pélagianisme durci, forcé peut-être dans le feu de la controverse par un partisan zélé (p. 184, 187). On voit l'intérêt de la question; on comprend aussi la 
difficulté, pour l'A., de trancher le problème qu'elle traite, face à un texte aussi mal assuré. Ces nombreux problèmes inhérents au texte utilisé appellent surtout une nouvelle édition qui, s'il n'est pas sûr qu'elle permettra de résoudre toute la question, aura du moins le mérite d'éliminer les variantes de tradition, qu'il n'y a pas lieu de commenter. En attendant que quelqu'un relève le gant, on pourra profiter d'une traduction (partielle) de ce commentaire, procurée par la même S. M., sur la base du texte de $A$, avec les indications des variantes de B: Pelagio, Commento all'epistola ai Romani - Commento alle epistole ai Corinzi, Roma, Città Nuova ("Testi patristici», 221), 2012.

\section{AUTEURS}

\section{JÉRÉMY DELMULLE}

Université de Paris-Sorbonne,

Katholieke Universiteit Leuven. 